\title{
ОПТИМИЗАЦИЯ МАРШРУТОВ ПРОКЛАДКИ МАГИСТРАЛЬНОГО ТРУБОПРОВОДА ДЛЯ ТРАНСПОРТИРОВКИ ГЕОРЕСУРСОВ
}

\author{
Токтошов Гулжигит Ысакович', \\ tgi_tok@rambler.ru
}

Юргенсон Анастасия Николаевна',

nastya@rav.sscc.ru

\author{
Мигов Денис Александрович', \\ mdinka@rav.sscc.ru
' Институт вычислительной математики и математической геофизики СО РАН, Россия, 630090, г. Новосибирск, пр. Академика Лаврентьева, 6.

\begin{abstract}
Актуальность исследования обусловлена необходимостью транспортировки добытого георесурса от месторождений до их переработки, с целью дальнейшего распределения между потребителями ресурсов различного назначения. В связи с этим актуальным является задача выбора маршрута прокладки магистрального трубопровода, осуществляющего транспортировку георесурсов от места добычи до мест их переработки. При этом должна быть обеспечена минимальность капитальных вложений и эксплуатационных затрат, а также надёжность функционирования магистрального трубопровода в условиях непредвиденных природно-техногенных воздействий.

Цель: предложить новый метод для прокладки магистрального трубопровода, учитывающий совместимость или несовместимость различных типов трубопроводного транспорта в одном техническом коридоре, а также надежность их функционирования.

Объекты: магистральный трубопровод, прокладываемый на заданной территории.

Методы: методы теории графов и теории гиперсетей; методы эволюционного синтеза; компьютерное моделирование процесса транспортировки георесурсов; дискретизация области размещения магистрального трубопровода.

Результаты. Предложен новый математический аппарат, основанный на модели теории гиперсетей, учитывающий иерархичность структуры проектируемого магистрального трубопровода. На основе гиперсетевого подхода получен новый метод прокладки магистрального трубопровода, учитывающий несовместимость различных типов ресурсов для прокладки в одном техническом коридоре. Разработан двухэтапный метод построения магистрального трубопровода, обеспечивающий минимальность капитальных вложений и эксплуатационных затрат. Кроме того, разработан модифицированный муравьиный алгоритм, обеспечивающий получение не только дешевого решения, но и достаточно надёжного по отношению к заданному порогу, при условии, что сбои происходят в первичной (физической) сети. В качестве показателя надёжности мы рассматриваем минимум среди всех парных вероятностей связности между каждым перерабатывающим пунктом и соответствующим источником. Полученные в ходе исследования численные эксперименты показывают применимость предложенных нами методов.
\end{abstract}

\section{Ключевые слова:}

Георесурсы, область размещения, транспортировка георесурсов, магистральный трубопровод, граф, гиперсеть, первичная сеть, вторичная сеть, надежность сети, эволюционные алгоритмы.

\section{Введение}

Роль трубопроводного транспорта в добыче и освоении полезных ископаемых (нефть, природный газ, подземные минеральные и пресные воды и т. п.) чрезвычайно высока. Трубопроводная транспортировка является основным и одним из дешёвых видов транспорта георесурсов от мест добычи на перерабатывающие пункты. При этом возникает задача поиска оптимального маршрута для прокладки магистрального трубопровода в областях со сложными природно-климатическими и инженерно-геологическими условиями, которые предопределяют направление прокладки и взаимное расположение трубопроводных систем. Для этой задачи необходимо находить достаточно приемлемый вариант решения, который позволяет максимизировать объемы добычи и получаемую прибыль, или минимизировать затраты на добычу, транспортировку и первичную переработку $[1,2]$.

Задачи, связанные с поиском маршрута прокладки, возникают и в других областях. Примера- ми подобных задач являются: поиск оптимальных маршрутов для прокладки инженерных сетей [3-6], размещение объектов на заданной территории $[7,8]$, использование ГИС-технологий для анализа и размещения линейных объектов $[9,10]$, применение сплайнов в оптимизации трассировки автомобильных дорог [11], задачи размещения логистических объектов [12] и систем электроснабжения [13], проектирование и реконструкция транспортных сетей [14], маршрутизация сетей обслуживания [15]. В этих работах объекты сетевой структуры, в т. ч. и магистральный трубопровод, рассматриваются как двумерные обьекты, размещенные на плоскости. Однако такое представление сетевых структур не достаточно соотвествует реальности.

При проектировании и строительстве сетевых объектов необходимо учитывать взаимодействие не менее чем двух взаимосвязанных подсистем. Так, например, магистральный трубопровод укладывается на участке земной поверхности, кото- 
рый, в свою очредь, становиться техногенным элементом этой среды. И наоборот, участок земли, по которому был проложен магистральный трубопровод, становиться частью трубопроводной системы. Таким образом, в данной работе магистральный трубопровод рассматривается как двухуровневая система, состоящая:

- из трассы (траншеи, коллекторы и т. п.) между заданными точками (источники и перерабатывающие пункты) на земной поверхности;

- магистрального трубопровода, укладываемого по соответствующим трассам, в зависимости от вида и назначения георесурса.

При выборе трассы магистрального трубопровода обобщающим критерием являются показатели капитальных вложений в сооружение трубопровода и эксплуатационные затраты в процессе его функционирования. Выбор направления трасс магистральных трубопроводов зависит от требований норм и технических условий на проектирование в части минимально допустимых расстояний от оси проектируемых линий (трубопроводов) до различных параллельно идущих коммуникаций, объектов и сооружений. Кроме того, выбор маршрута прокладки магистрального трубопровода на заданной территории затруднён большим количеством специфических природных условий, предопределяющих объем работ, их стоимость и надёжность эксплуатации. К таким условиям относятся, прежде всего, сложный пересеченный рельеф местности с большим диапазоном относительных и абсолютных отметок, нежелательные физико-геологические явления (камнепады, снежные лавины, землетрясения, оползни и т. п.) и сложные метеорологические условия.

\section{О математической модели магистрального трубопровода}

Для моделирования различных сетевых объектов обычно используются графы и гиперграфы. Как уже упоминалось выше, структура проектируемого магистрального трубопровода представляет собой двухуровневую взаимосвязанную сеть трасса и магистральный трубопровод, образующие иерархические структуры. В таком случае применение аппарата теории графов и гиперграфов не позволяет учесть реализацию трубопроводной системы по выбранным трассам. Поэтому в большинстве случаев вместо них используют другие сетевые модели [3, 4, 16-20]: вложенные графы [16], многоуровневые комплексные сети [17], гиперсети [18-20], а также другие объекты.

В настоящей работе в качестве математической модели для структуры магистрального трубопровода используется гиперсеть, поскольку в ней учитывается реализация вложения одной структуры в другую. В данном случае это реализация магистрального трубопровода по выбранным маршрутам, отмеченным на этапе проектирования. Гиперсеть, в отличие от графов и гиперграфов, учитывает взаимодействие двух подсистем, таких как трас- са и магистральный трубопровод, а также взаимозависимость характеристик их элементов. Так, например, от вида и назначения транспортируемого ресурса (соответственно и от типа трубопровода) зависят стоимостные характеристики области размещения (аренда земельного участка, рытье транше, подготовительные работы, строительство опоры и т. п.). От категории местности (горная, холмистая, или равнинная) сильно зависят стоимостные характеристики будущего трубопровода. Некоторые наши результаты по применению данного подхода были приведены в работах [20-22].

Приведём наиболее общее определение гиперсети [18-20], несмотря на то, что для рассматриваемой задачи достаточно и более простого определения [21].

Oпределение. Гиперсеть $H N=(X, Y, R ; P, F, W)$ включает следующие объекты:

$X=\left(x_{1}, x_{2}, \ldots x_{n}\right)-$ множество узлов (вершин); $V=\left(v_{1}, v_{2}, \ldots v_{g}\right)-$ множество трасс; $R=\left(r_{1}, r_{2}, \ldots r_{m}\right)-$ множество трубопроводов; $P: V ? X^{2}$ - отображение, сопоставляющее каждому элементу $v \in V$ множество вершин $P(v) \subseteq \rightarrow X$. Тем самым отображение $P$ определяет граф первичной сети $P N=(X, Y)$; $F: R \rightarrow 2^{V}$ - отображение, сопоставляющее каждому элементу $r \in R$ множество трасс $(r)$, образующих простой маршрут в графе $P N=(X, V)$. Множество всех маршрутов $F(r)$, отображающее каждому ребру $r \in R$ графа $S N$ единственный маршрут в графе $P N$, назовем вложением графа $S N$ в $P N$; $W: R \rightarrow 2^{V(F(r))}$ - отображение, сопоставляющее каждому элементу $r \in R$ множество вершин $W(r) \subseteq P(F(r))$ в графе $P N$, где $P(F(r))=Y-$ множество вершин в $P N$, инцидентных трассам $F(r) \subseteq V$. Отображение $W$ определяет граф вторичной сети $S N=(Y, R)$.

Из определения гиперсети следует, что математическая модель возможных трасс соответствует графу первичной сети $P N=(X, V)$, а структура магистрального трубопровода - графу вторичной сети $S N=(Y, R)$. Взаимодействие этих сетей определяется гиперсетью $H N$.

\section{Совместимость различных типов ресурсов для прокладки в одном направлении}

Рассмотрим возможность совмещенной прокладки параллельно идущих трубопроводов в одном техническом сооружение (траншее, коллекторе и т. п.) Необходимо учесть совместимость или несовместимость этих коммуникаций и нормативное расстояние между трубопроводами различного назначения.

Прокладка инженерных сетей по выбранной трассе (траншея, коллектор и т. п.) осуществляется строго с соблюдением установленных норм и правил, касающихся взаимного расположения сетей различного назначения. При этом должны учитываться минимально допустимые расстояния между различными инженерными сетями, прокладываемыми в одном направлении. В зависимости от вида, назначения и направления прокладки инже- 
нерные сети могут быть проложены как совмещённо, так и раздельно. Отметим, что совмещенная прокладка сетей различного назначения в одном коллекторе допускается исключительно для совместимых (нейтральных) по механическим и электромагнитным воздействиям друг на друга сетей.

Так, например, в одном коллекторе заданного размера могут быть проложены идущие в одном направлении тепловые сети, напорные водопроводы и канализация, свыше десяти кабелей связи и силовых кабелей напряжением до 10 кВ. Не разрешается совместная прокладка газопроводов и трубопроводов с горючими веществами.

Таким образом, прокладка сетей инженерных коммуникаций связана с определением пространственного расположения всех элементов различных инженерных коммуникаций. Поиск оптимального варианта их размещения в одном коллекторе требует анализа множества этих вариантов, каждый из которых должен быть проверен на соответствие ограничениям математической модели. Эти ограничения включают в себя условия непересечения несовместимых инженерных коммуникаций, их взаимного расположения, и другие ограничения [23].

\section{Постановка задачи}

$D=\{(x, y) \mid a<x<b, c<y<d\}-$ прямоугольная картографируемая область, на которой предполагается строительство магистрального трубопровода;

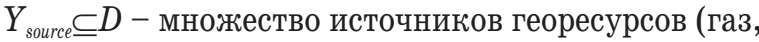
нефть, пресная вода и т. д.); $Y_{\text {station }} \subseteq D-$ множество пунктов переработки.

Множество вершин $X$ графа первичной сети $P N=(X, V)$ включает в себя множество $\mathrm{Y}=Y_{\text {source }} \cup Y_{\text {station }}$

Пусть в графе $P N=(X, V)$ заданы следующие характеристики: $l(v)$ - длина ветви $v \in V ; a(v)$ - стоимость земли (аренда, налог и т. д.) на участке $v \in V$ предполагаемой трассы; $b(v)$ - стоимость подготовительных и строительных работ (копка траншеи, строительство коллекторов, тоннелей и опор и т. п.) на участке $v \in V$ предполагаемой трассы; $\gamma_{1}-$ коэффициент дисконтирования строительных затрат для приведения экономических показателей разных лет к сопоставимым по времени величинам).

Пусть в графе вторичной сети $S N=(Y, R)$ заданы следующие параметры: $\rho(r)=\sum_{v \in F(r)} l(v)$ - длина трубопровода $r \in R$, проходящего по ветвям $v \in F(r)$ предполагаемой трассы; $c(r)$ - стоимость строительства и монтажа самого трубопровода $r \in R$, coединяющего соответствующие пары узлов из $Y ; \gamma_{2}-$ коэффициент дисконтирования стоимости трубопровода; $d_{v}(r)$ - стоимость эксплуатации трубопроводов на участках $v \in F(r)$.

Пусть $T$ - множество типов ресурсов, предаваемых через магистральные трубопроводы. Для каждого ребра $r \in R$ зададим тип type $(r) \in T$. Тип ресурса однозначно определяет тип магистрального трубопровода.
Как было сказано выше, разные типы ресурсов могут быть несовместимы друг с другом для их прокладки в одном техническом коридоре.

Пусть бинарное отношение $C T \subseteq T \times T$ определяется правилом: если $\left(t_{1}, t_{2}\right) \in C T$, то эти типы ресурсов могут быть проложены в одном и том же технологическом коридоре, т. е. два трубопровода различных типов могут быть проложены в одной ветви первичной сети.

Пусть $\operatorname{MinCT}\left(t_{1}, t_{2}, \ldots, t_{h}\right)$ - минимальное число непересекающихся подмножеств, на которое можно разделить типы ресурсов $\left\{t_{1}, t_{2}, \ldots, t_{h}\right\}$ с учетом их совместимости.

Например, если существуют типы ресурсов $\left\{t_{1}, t_{2}, t_{3}\right\}$, такие что $\left(t_{1}, t_{2}\right),\left(t_{2}, t_{3}\right) \in C T$, но $\left(t_{2}, t_{3}\right) \notin C T$, тогда MinCT $\left(t_{1}, t_{2}, t_{3}\right)=2$, поскольку эти типы ресурсов могут быть разделены на два подмножества $\left\{t_{1}, t_{2}\right\}$ и $\left\{t_{3}\right\}$.

В общем случае задача поиска маршрутов прокладки магистрального трубопровода может быть сформулирована как задача поиска трасс для прокладки трубопроводов между заданных пар точек из $Y=Y_{\text {source }} \cup Y_{\text {station }}$, обеспечивающих минимальность затрат на построение и последующую эксплуатацию магистрального трубопровода.

Постановка задачи: построить гиперсеть $H N$, т. е. каждому ребру $r \in R$ графа вторичной сети найти соответствующий маршрут в графе первичной сети $P N$ таким образом, чтобы выполнялись все условия и ограничения, накладываемые на проектируемый тип трубопровода, причем следующий функционал должен иметь минимальное значение:

$$
\begin{gathered}
Q(H N)=\sum_{v \in V^{\prime}}\left(a(v)+b(v) \gamma_{1}\right) \rho(v) \operatorname{MinCT}(v)+ \\
+\sum_{r \in R}\left(c(r)+\sum_{v \in F(r)} d(r) \gamma_{2}\right) \rho(r),
\end{gathered}
$$

где подмножество $V^{\prime} \subseteq V$ определяется следующим образом: для каждого элемента $v \in V^{\prime}, \exists r \in R$ такое, что $v \in F(r)$. Пусть $v \in V^{\prime}$ и $v \in F\left(r_{i}\right), i=1, \ldots, l\left(r_{1}, \ldots, r_{l} \in R\right)$, тогда $\operatorname{MinCT}(v)=\operatorname{MinCT}\left(\operatorname{type}\left(r_{1}\right), \ldots, \operatorname{type}\left(r_{l}\right)\right)$.

\section{Двухэтапный алгоритм}

В данном разделе предлагается двухэтапный алгоритм решения поставленной задачи. Сначала находится начальное приближенное решение, затем оно улучшается.

Для поиска начального решения используется «жадный» алгоритм и классический алгоритм Флойда.

Для алгоритмов Флойда и Дейкстры стоимость ветви $v \in V$ на графе первичной сети $P N$ равна $\left(a(v)+b(v) \gamma_{1}+c(r)+d(r) \gamma_{2}\right) \rho(v)$. Для простоты будем считать $\forall r \in R: c(r)+d(r) \gamma_{2}=$ const.

Ниже будут описаны шаги предлагаемого алгоритма.

Алгоритм 1 («жадный»)

Шаг 1. Разделить типы ресурсов на непересекающиеся подмножества. 
Шаг 2. Выбрать подмножество совместимых типов $T^{\prime}$ с наибольшим количеством ребер (для которых еще не найдено вложение в первичную сеть).

Шаг 3. Найти все кратчайшие пути $\left(y_{i}, y_{i}\right)$, $i, j=1, \ldots, n, i \neq j$ (существует ребро $r$ ) в графе первичной сети $P N=(X, Y)$ с помощью алгоритма Флойда. Паре вершин $\left(y_{i}, y_{j}\right)$ соответствует ребро $r$ во вторичной сети и type $(r) \in T^{\prime}$.

Выбрать среди них путь минимальной стоимостью.

Осуществить вложение выбранного ребра $r$ по кратчайшему пути $\left(y_{i}, y_{j}\right)$ в графе $P N$.

Для всех $v \in F(r)$ в графе первичной сети $P N a(v):=0, b(v):=0$ (стоимость ветви равна нулю для прокладки остальных ребер).

Повторить Шаг 3 пока для всех ребер $r \in R$ вторичной сети, таких что type $(r) \in T^{\prime}$, не будут найдены и реализованы кратчайшие пути.

Шаг 4. Присвоить изначальные значения $a(v)$ и $b(v)$, которые обнулены на предыдущем шаге. Если не $T^{\prime} \neq 0$, переходим на Шаг 2 .

Отметим, что жадный алгоритм является приближенным, поэтому полученные решения не всегда оптимальны. Можно улучшить решение, найдя новый маршрут $r \in R$. Например, новый маршрут $F(r)$ дешевле для выбранного $r \in R$, если он включает в себя большее количество ветвей $v \in F(r)$ с нулевой стоимостью (ветви, которые уже используется для других ребер $r \in R$ ).

Можно упорядочит ребра по некоторому правилу, например, по числу наиболее редко используемых ветвей. Отметим, что в среднем полученные результаты сильно не зависят от используемого метода упорядочения [21].

Алгоритм 2 (Модифицированный)

ШІаг 1. Упорядочить $r \in R$ по убыванию их веса (получим список $\left\{r_{i}\right\}$ ).

Шаг 2. «Удалить» $r_{i}$ из гиперсети $H N$ согласно списку, т. е. $\forall v_{k} \in F\left(r_{i}\right)$, восстановить начальные значения $a\left(v_{k}\right)$ и $b\left(v_{k}\right)$, если $v_{k}$ не входит в другие ребра.

Найти новый кратчайший путь для $r_{i}$ в графе $P N$ алгоритмом Дейкстры.

Для нового ребра $r_{i}: a(v):=0, b(v):=0$ для всех $v \in F\left(r_{i}\right)$ в графе $P N$.

Повторить Шаг 2 пока все ребра $\left\{r_{i}\right\}$ из списка не будут обновлены.

Можно использовать несколько итераций Алгоритма 2 для поиска лучших результатов.

\section{Численные эксперименты}

В качестве первичной сети $P N$ была взята сетка размерностью $10 \times 10$ вершин. Количество ребер в $S N$ для укладки в $P N$ варьировалось от 10 до 800 (ось абсцисс на рис. 1).

Источники ресурсов и потребители произвольно размещались в сетке. Узел сетки может содержать несколько источников и потребителей. Каждому ребру случайно присваивается один из трех типов (набор типов ресурсов взят из примера в постановке задачи).
Случайное размещение выполняется 10 раз для заданного значения $M$. Найденная стоимость укладки усредняется.

На рис. 1 показана нормированная стоимость (т. е. находится минимальная стоимость и на нее делится все полученные значения). Из рисунка видно, что «жадный» алгоритм обеспечивает лучший результат для небольшого числа ребер, а для большого числа ребер более приемлемым является алгоритм Флойда.

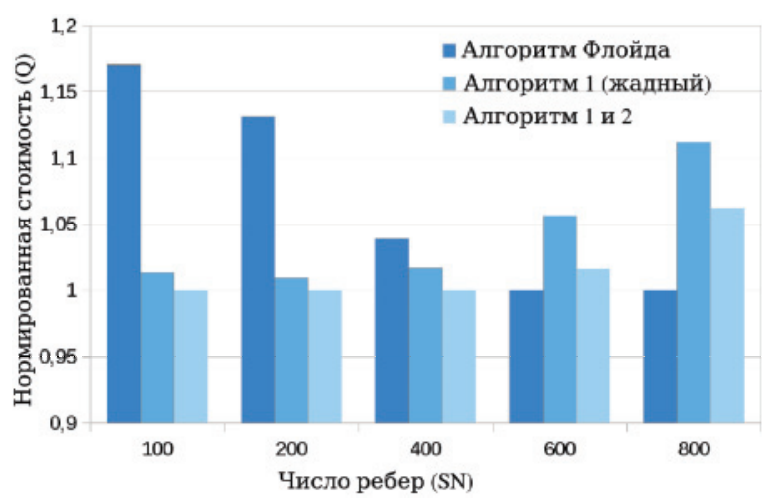

Рис.1. Численные результаты: норлированная стоилость для решетки $10 \times 10$, количество ребер от 10 до 800

Fig. 1. Numerical results: normalized cost for the $10 \times 10$ grid, the number of edges from 10 up to 800

\section{Прокладка магистрального трубопровода \\ с учетом заданного уровня надёжности}

В этом разделе мы модифицируем ранее предложенный алгоритм для нахождения не только самого дешевого решения, но и достаточно надёжного. Отметим, что анализ надежности гиперсетей не является новой задачей $[23,24]$. В качестве меры надежности рассматривается гиперсетевой аналог двухтерминальной надежности, при условии, что в первичной сети происходят сбои, а узлы во вторичной сети должны быть надежно соединены. В этом случае мы проектируем инженерную сеть, в которой каждый потребитель должен быть связан с необходимыми поставщиками с вероятностью не менее заданного порога надежности $0<R \leq 1$. Для решения этой проблемы используем методом алгоритма колонии муравьев. В отличие от существующих методов оптимизации предлагаемый метод позволяет получить оптимальный вариант размещения инженерной сети на заданной территории по критерию «надежность функционирования будущей сети и минимальность затрат на её строительство и эксплуатацию».

Как правило, для моделирования систем сетевой структуры с ненадёжными элементами используют аппарат случайных графов, а надёжность сети определяется как некоторая мера связности случайного графа. Наиболее распространенным показателем сетевой надёжности является вероятность связности сети, т. е. вероятность того, что все узлы в сети могут устанавливать соединение друг с другом. Задача расчета вероятности связности сети NP 
трудна [25]. Тем не менее, можно осуществлять расчет и делать достоверные оценки надежности для сетей с использованием современных высокопроизводительных суперкомпьютеров [26-28], а также принимая во внимание некоторые особенности реальных сетевых структур [29-31].

Еще одна хорошо известная мера надёжности сети - это двухтерминальная надежность, т. е. вероятность связности двух выделенных узлов сети (терминалов) [29]. Средняя парная связность $[30,31]$ представляет собой среднюю двухтерминальную надежность сети среди всех пар узлов. Этот показатель описывает надёжность сети с точки зрения соединения между двумя произвольными узлами, даже если сеть в целом не связна.

Будем считать, что ветви (ребра первичной сети) подвержены случайным отказам, происходящим независимо друг от друга с заданными вероятностями $p_{i}, 1 \leq i \leq g$.

Надежность ребра вторичной сети $r \in R$ определим как

$$
R_{r}(H N)=\prod_{v \in F(r)} p(v) .
$$

Если для ребра $r \in R$ путь $F(r)$ имеет конечные точки $a$ и $b$ и никакие другие рёбра не связывают эти точки, мы будем использовать обозначение $R_{a b}(H N)$ вместо $R_{r}(H N)$. Если для узлов $a$ и $b$ существует более одного такого ребра, это обозначение $R_{a b}(H N)$ используется для того ребра, значение надежности которого максимально. Однако в рассматриваемой задаче (1) требуется соединить поставщиков с потребителями с минимальной стоимостью, поэтому нас не интересуют подобные случаи как приводящие к дорогим и заведомо неоптимальным решениям.

Введем в рассмотрение меру надежности $R$ $(H N)$ для гиперсети $H N$ в целом, с учетом того, что в первичной сети происходят сбои, но при этом все потребители должны быть связаны с необходимыми им поставщиками:

$$
R(H N)=\min \left\{R_{a b}(H N)\right\}, a \in Y_{\text {source }}, b \in Y_{\text {station }} \text {. }
$$

Таким образом, $R(H N)$ является минимумом среди всех двухтерминальных надежностей $R_{a b}(H N)$, где $b$ - это перерабатывающий пункт, $a-$ источник ресурса.

Основываясь на ранее описанном двухэтапном алгоритме, мы предлагаем новый алгоритм прокладки трубопроводов между заданным точками на графе первичной сети с ограничением на их надёжность. Предполагается, что задан порог надёжности $0<R_{0} \leq 1$. Задача состоит в том, чтобы построить гиперсеть $H N$ минимальной стоимости и при этом достаточно надёжную, т. е. с условием $R(H N)>R_{0}$

\section{Алгоитм муравьиной колонии}

В случае ограничения на надёжность удобно использовать алгоритм муравьиной колонии [32]. Для каждого ребра мы создаем муравьев, действующих по следующим правилам:
- вероятность перехода муравья в следующую вершину на итерации $t$ определяется как:

$$
P_{i j}(t)=\frac{\left[\tau_{i j}(t)\right]^{\alpha}\left[v_{i j}\right]^{\beta}}{\sum\left[\tau_{i j}(t)\right]^{\alpha}\left[v_{i j}\right]^{\beta}} ;
$$

- каждый муравей $l_{i}(0<l<L)$ имеет метку TimeToLive TTL $\left(l_{i}\right)$ :

$$
\operatorname{TTL}\left(l_{i}\right)=\prod_{v \in \operatorname{Path}\left(l_{i}\right)} p(v),
$$

где $\operatorname{Path}\left(l_{i}\right)$ - это путь для муравья $l_{i}$. При этом если $T T L\left(l_{i}\right)<R_{0}$, то муравей $l_{i}$ умирает.

- для каждого ребра муравей откладывает феромон на каждой ветви $\Delta \tau_{i j}(t)=Q / \operatorname{leght}(t)$;

- в наборе наиболее частых маршрутов находится и фиксируется минимум, стоимость ветвей первичной сети уменьшается.

\section{Численные эксперименты}

На рис. 2 представлены численные результаты предложенного алгоритма. Для надёжности были заданы следующие значения: $p_{i}=0,99,1 \leq i \leq g$, $R_{0}=0,9$. В качестве графа первичной сети $P N$ взята сетка размерность $10 \times 10$. Параметры алгоритма муравьиной колонии следующее: $\alpha:=1 ; \beta:=3$; $\tau_{0}:=1 ; q:=50$.

Количество ребер в $S N$ для укладки в $P N$ варьировалось от 10 до 100 (ось абсцисс на рис. 2).

Ось ординат - стоимость прокладки гиперсети $Q(H N)$. Сравнивались результаты работы «жадного» алгоритма и алгоритма муравьиной колонии. Из рисунка видно, что муравьиный алгоритм дает лучший результат для небольшого числа ребер. Когда ребер много, результаты работы алгоритмов почти одинаковы. Однако при этом алгоритм Флойда находит решение значительно быстрее.

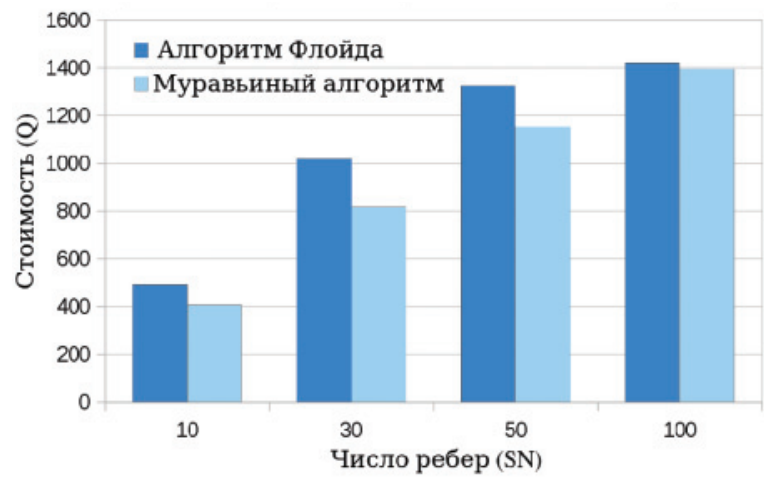

Pис.2. Численные результаты: стоимость для решетки $10 \times 10$ для количества ребер от 10 до 100

Fig. 2. Numerical results: costs for $10 \times 10$ grid, the number of edges from 10 up to 100

\section{Заключение}

Изучен новый подход к проектированию магистрального трубопровода для доставки и переработки георесурсов. В отличие от классических моделей, в предложенной модели мы рассматриваем природно-техническую систему «земельный уча- 
сток - магистральный трубопровод» в рамках одной математической модели. Как результат - предлагается новый метод нахождения маршрутов для прокладки трубопроводов между источниками георесурсов и пунктами их переработки с минимизацией затрат на укладку и техническое обслуживание с учетом заданных ограничений. Этот метод позволяет найти решение с учетом совместимости различных типов ресурсов для размещения их в одной и той же трассе.

Для обеспечения надёжности проектируемой сети предлагается метод получения не только де-

\section{СПИСОК ЛИТЕРАТУРЫ}

1. Смирнов П.В. Предварительные результаты ревизии минерально-сырьевой базы опал-кристобалитовых пород в среднем Зауралье // Известия Томского политехнического университета. Инжиниринг георесурсов. - 2017. - Т. 328. - № 4. C. 28-37.

2. Полубоярцев Е.Л., Благовисный П.В., Исупова Е.В. Трубопроводный транспорт нефти и газа. - Ухта: УГТУ, 2014. - 39 с.

3. Network Design and Cost Optimization for Label Switched Multilayer Photonic IP Networks / S. Kaneda, T. Uyematsu, N. Nagatsu, K. Sato // J. IEEE Journal on Selected Areas in Communications. - 2005. - V. 23 (8). - P. 1612-1619.

4. Belotti P., Malucelli F. Row-Column Generation for Multilayer Network Design // Int. Network Optimization Conf. - Lisbon, Portugal, 2005. - P. 422-427.

5. Лотарев Д.Т. Неформальные описательные модели транспортных коммуникаций, транспортных сетей и территорий в задаче 0 прокладке путей и коммуникаций // Труды ИСА РАН. 2009. - T. 46. - C. 259-273.

6. Мелькумов В.Н., Кузнецов И.С., Кузнецов Р.Н. Определение оптимального маршрута трассы газопровода на основе карт стоимости влияющих факторов // Научный вестник ВГАСУ. Строительство и архитектура. - 2009. - № 1 (13). - С. 21-27.

7. Забудский Г.Г., Веремчук Н.С. Алгоритм приближенного решения задачи Вебера на линии с запрещенными зонами // Дискретн. анализ и исслед. опер. - 2016. - Т. 23. - № 1. C. 82-96.

8. Davydov I., Kochetov Y., Dempe S. Local search approach for the competitive facility location problem in mobile networks // International Journal of Artificial Intelligence. - 2018. - V. 16 (1). P. $130-143$.

9. Сарычев Д.С. Современные информационные системы для инженерных сетей // Вестник ТГУ. - 2003. - № 280. C. $358-361$.

10. Скворцов А.В. Разработка геоинформационных и инженерных систем на факультете информатики и в 000 «Индорсофт» // Вестник ТГУ. - 2003. - № 280. - С. 346-349.

11. Бойков В.Н., Шумилов Б.М. Сплайны в трассировании автомобильных дорог. - Томск: ЦНТИ, 2001. - 164 с.

12. Лемперт А.А. Математическая модель и программная система для решения задачи размещения логистических объектов / А.А. Лемперт, А.Л. Казаков, Д.С. Бухаров // Управление большими системами. - 2013. - № 41. - С. 270-284.

13. Косяков С.В., Новосельцева С.С., Садыков А.М. Мягкие вычисления в построении карт зонирования территорий по параметрам систем энергоснабжения // Состояние и перспективы развития электротехнологии: материалы международной научно-технической конференции. - Иваново, 2013. - С. 338-341.

14. Прилуцкий М.Х., Афраймович Л.Г. Распределение ресурсов в иерархических системах транспортного типа. - Нижний Новгород, 2007. - 80 c. шевого решения, но и достаточно надёжного по отношению к заданному порогу, при условии, что сбои происходят в первичной (физической) сети. В качестве показателя надёжности рассматривается минимум среди всех парных вероятностей связности между каждым перерабатывающим пунктом и соответствующим источником. Приведенные численные эксперименты показывают применимость предложенных методов.

Работа поддержана Программой фундаментальных исследований ИВМиМГ СО РАН № 0315-2016-0006 и проектом РФФИ № 18-07-00460

15. Ерзин А.И., Кочетов Ю.А. Задачи маршрутизации. - Новосибирск, 2014. -95 с.

16. Poulovassilis A., Levene M. A Nested-Graph Model for the Representation and Manipulation of Complex Objects // J. ACM Trans. Inf. Syst. -1994. - V. 12. - P. 35-68.

17. Kurant M., Thiran P. Layered Complex Networks // J. Phys. Rev. Lett. - 2006. - V. 96. - 138701-1-138701-4.

18. Попков В.К. 0 моделировании городских транспортных систем гиперсетями // Автоматика и телемеханика. - 2011. T. 72. - № 6. - C. 179-189.

19. Sokolova 0.D., Yurgenson A.N. Using Graph, Hypergraph and Hypernet Models for Network Analysis Problems // $7^{\text {th }}$ IEEE Forum on Strategic Technology. - Tomsk, 2012. - P. 1-4.

20. Toktoshov G.Y., Monakhov 0.G. Models and Algorithms of Evolutionary Synthesis for Optimization of Engineering Networks // Proc. of International Multi Conference on Engineering, Computer and Information Sciences. IEEE SIBIRCON-2017. - Novosibirsk, Russia, 2017. - P. 167-171.

21. Toktoshov G.Y., Yurgenson A.V., Migov D.A. Design of Utility Network Subject to Reliability Constraint // Proc. of International Multi-Conference on Engineering, Computer and Information Sciences. IEEE SIBIRCON-2017. - Novosibirsk, Russia, 2017. P. 172-175.

22. СНиП 2.07.01-89* Градостроительство. Планировка и застройка городских и сельских поселений. - М.: Горстрой, 2016. -89 c.

23. Rodionov A.S., Rodionova 0.K. Random Hypernets in Reliability Analysis of Multilayer Networks // J. Lecture Notes in Electrical Engineering. - 2015. - V. 343. - P. 307-315.

24. Rodionov A.S., Rodionova 0.K. Using Random Hypernets for Reliability Analysis of Multilevel Networks // 1st Int. Conf. on Mathematical Methods and Computational Techniques in Science and Engineering (MMCTSE 2014). Ser. Mathematical Methods in Science and Engineering. - Athens, Greece, 2014. - P. 119-121.

25. Colbourn Ch.J. The Combinatorics of Network Reliability. -New York: Oxford University Press, 1987. - 160 p.

26. Martnez S.P., Calvino B.O., Rocco S.C.M. All-Terminal Reliability Evaluation through a Monte Carlo Simulation Based on an MPI Implementation // European Safety and Reliability Conference: Advances in Safety, Reliability and Risk Management (PSAM 2011/ESREL 2012). - Helsinki, 2012. - P. 1-6.

27. Migov D.A., Rodionov A.S. Parallel Implementation of the Factoring Method for Network Reliability Calculation // ICCSA-2014. LNCS. - Heidelberg: Springer, 2014. - V. 8584 (4). - P. 654-664.

28. Nesterov S.N., Migov D.A. Parallel Calculation of Diameter Constrained Network Reliability // PACT-2017. LNCS. - Heidelberg: Springer, 2017. - V. 10421. - P. 473-479.

29. Shooman A.M., Kershenbaum A. Exact Graph-Reduction Algorithms for Network Reliability Analysis / IEEE Global Telecommunications Conference GLOBECOM' 91. - New York: IEEEP Press, 1991. - P. 1412-1420. 
30. Rodionov A.S. Cumulative Estimated Values of Structural Networks Reliability Indices and their Usage // IEEE Conf. on Dynamics of Systems, Mechanisms and Machines. - Omsk, Russia: IEEE Press, 2016. - P. 1-4.

31. Родионов А.С. Кумулятивные оценки показателей структурной надежности сети и их использование // Проблемы информатики. - 2017. - № 34. - С. 15-24.
32. Dorigo M. Swarm Intelligence, Ant Algorithms and Ant Colony Optimization // Reader for CEU Summer University Course «Complex System». - Budapest, Central European University, 2001. - P. 1-38.

\section{Информация об авторах}

Токтошов Г.Ы., кандидат технических наук, научный сотрудник лаборатории системного моделирования и оптимизации Института вычислительной математики и математической геофизики СО РАН.

Юргенсон А.Н., кандидат физико-математических наук, научный сотрудник лаборатории системного моделирования и оптимизации Института вычислительной математики и математической геофизики СО РАН.

Мигов Д.А., кандидат физико-математических наук, старший научный сотрудник лаборатории системного моделирования и оптимизации Института вычислительной математики и математической геофизики СО PAH. 
UDC 519.17: 621.644.07

\title{
OPTIMIZATION OF ROUTES FOR LAYING TRUNK PIPELINE TO TRANSPORT GEO-RESOURCES
}

\author{
Gulzhigit Y. Toktoshov', \\ tgi_tok@rambler.ru
}

\section{Anastasiya N. Yurgenson', nastya@rav.sscc.ru}

\author{
Denis A. Migov', \\ mdinka@rav.sscc.ru \\ 1 Institute of Computational Mathematics and Mathematical Geophysics of SB RAS, \\ 6, Academician Lavrentyev avenue, Novosibirsk, 630090, Russia.
}

The relevance of the research is caused by necessity to transport geo-resources from extraction sites to processing sites for further distribution of different resources among consumers. In this regard, the task of choosing the routes for laying a trunk pipeline to transport geo-resources from extraction sites to processing sites is relevant. At the same time, it is necessary to provide a minimum spending (capital investment and operating costs) and reliability of the trunk pipeline operation in conditions of unforeseen natural and technogenic impacts.

The main aim of the research is to propose a new method for laying a trunk pipeline, taking into account the compatibility or incompatibility of different geological resources types for laying in the same technical corridor, as well as a reliability of a trunk pipeline.

Object: trunk pipeline laying on a given area.

Methods: methods of graph theory and theory of hypernets; methods of evolutionary synthesis; computer simulation of geo-resources transportation; discretization of the trunk pipeline location area.

Results. The authors have proposed a new mathematical apparatus based on the hypernet theory, taking into account the hierarchy of the structure of a designed trunk pipeline. On the basis of the hypernet approach, a new method for laying a trunk pipeline is obtained, taking into account the incompatibility of different types of resources for laying in the same technical corridor. A two-stage method is developed for designing a trunk pipeline ensuring minimum capital investment and operating costs. In addition, the authors developed a modified ant colony algorithm which provides not only a cheap solution, but reliable enough with respect to a given threshold in assumption that failures occur in the primary (physical) network. As a measure of reliability we consider the minimum among all pair probabilities of connectivity between each processing point and the corresponding source. The numerical experiments show the applicability of the methods proposed.

\section{Key words:}

Geo-resources, area of location, transportation of geo-resources, trunk pipeline, graph, hypernet, primary network, secondary network, network reliability, evolutionary algorithms.

The research was supported by the Basic Research Program of the ICMMG SB RAS No.0315-2016-0006 and the RFBR project No. 18-07-00460.

\section{REFERENCES}

1. Smirnov P.V. Preliminary results of the revision of the mineral resource base of opal-cristobalite rocks in the Middle Trans-Urals. Bulletin of the Tomsk Polytechnic University. Geo Assets Engineering, 2017, vol, 328, no. 4, pp. 28-37. In Rus.

2. Poluboyartsev E.L., Blagovisny P.V., Isupova E.V. Truboprovodny transport nefti i gaza [Pipeline transportation of oil and gas]. Ukhta, UGTU Publ., 2014. 39 p.

3. Kaneda S., Uyematsu T., Nagatsu N., Sato K. Network Design and Cost Optimization for Label Switched Multilayer Photonic IP Networks. IEEE Journal on Selected Areas in Communications, 2005, vol. 23 (8), pp. 1612-1619

4. Belotti P., Malucelli F. Row-Column Generation for Multilayer Network Design. Int. Network Optimization Conf. Lisbon, Portugal, 2005. pp. 422-427.

5. Lotarev D.T. Neformalnye opisatelnye modeli transportnykh kommunikatsy, transportnykh setey i territoriy v zadache 0 prokladke putey i kommunikatsy [Unformal Descriptive Models of Transport Communications, Transport Networks and Territory in the Problem of Construction of Ways and Communications]. Trudy instituta sistemnogo analiza Rossiyskoy akademii nauk [Proceedings of the Institute of Informatics Systems of the Russian Academy of Sciences]. Moscow, 2009. Vol. 46, pp. 259-273.
6. Melkumov V.N., Kuznetsov I.S., Kuznetsov R.N. Determination of the Optimal Route of the Gas Pipeline on the Basis of the Factors Affecting the Value of Cards. Construction and Architecture, 2009, vol. 1 (13), pp. 21-27. In Rus.

7. Zabudskii G.G., Veremchuk N.S. An algorithm for finding an approximate solution to the Weber problem on a line with forbidden gaps. Journal of Applied and Industrial Mathematics, 2016, vol. 23, no. 1, pp. 82-96. In Rus.

8. Davydov I., Kochetov Y., Dempe S. Local search approach for the competitive facility location problem in mobile networks. International Journal of Artificial Intelligence, 2018, vol. 16 (1), pp. $130-143$.

9. Sarychev D.S. Modern Information Systems for Utility Networks. Bulletin of Tomsk State University, 2003, no. 280, pp. 358-361. In Rus.

10. Skvortsov A.V. Development of Geographic Information and Utility Systems at the Faculty of Informatics in Ltd. "Indorsoft». Bulletin of Tomsk State University, 2003, no. 280, pp. 346-349. In Rus.

11. Boikov V.N., Shumilov B.M. Splayny v trassirovanii avtomobilnykh dorog [Splines in Routing of Roads]. Tomsk, TsNTI Publ., $2001.164 \mathrm{p}$.

12. Lempert A.A., Kazakov A.A., Bukharov D.S. Mathematical Model and Software System for the Solution of Location Problems of 
Logistics Objects. Management of big systems, 2013, no. 41, pp. 270-284. In Rus.

13. Kosyakov S.V., Novoseltseva S.S., Sadykov A.M. Myagkie vychisleniya v postroenii kart zonirovaniya territoriy po parametram sistem energosnabzheniya [Soft Computing in Mapping Zoning by Parameters of Power Supply Systems]. Sostoyanie i perspektivy razuitiya elektrotekhnologii. Materialy mezhdunarodnoy nauch no-tekhnicheskoy konferentsii [International scientific-technical conference. State and prospects of development of electrotechnology]. Ivanovo, 2013. pp. 338-341.

14. Prilutskiy M.Kh., Afraiymovich L.G. Raspredelenie resursov v ierarhicheskikh sistemakh transportnogo tipa [Distribution of resources in transport-type hierarchical systems]. Nizhny Novgorod, $2007.80 \mathrm{p}$.

15. Erzin A.I., Kochetov Yu.A. Zadachi marshrutizatsii [Routing problems]. Novosibirsk, $2014.95 \mathrm{p}$.

16. Poulovassilis A., Levene M. A Nested-Graph Model for the Representation and Manipulation of Complex Objects. ACM Trans. Inf. Syst., 1994, vol. 12, pp. 35-68.

17. Kurant M., Thiran P. Layered Complex Networks. Phys. Rev. Lett., 2006, vol. 96, pp. 138701-1-138701-4.

18. Popkov V.K. On Modeling City Traffic Systems with Hypernetworks. Automation and Remote Control, 2011, vol. 72 (6), pp. 179-189. In Rus.

19. Sokolova O.D., Yurgenson A.N. Using Graph, Hypergraph and Hypernet Models for Network Analysis Problems. $7^{\text {th }}$ IEEE Forum on Strategic Technology. Tomsk, 2012. pp. 1-4.

20. Toktoshov G.Y., Monakhov 0.G. Models and Algorithms of Evolutionary Synthesisfor Optimization of Engineering Networks. Proc. of International Multi Conference on Engineering, Computer and Information Sciences, IEEE SIBIRCON-2017. Novosibirsk, Russia, 2017. pp. 167-171.

21. Toktoshov G.Y., Yurgenson A.V., Migov D.A. Design of Utility Network Subject to Reliability Constraint. Proc. of International Multi-Conference on Engineering, Computer and Information Sciences, IEEE SIBIRCON-2017. Novosibirsk, Russia, 2017. pp. $172-175$.

22. SNiP 2.07.01-89*. Gradostroitelstvo. Planirovka i zastroyka gorodskikh $i$ selskikh poseleniy [Building Norms and Rules
2.07.01-89*. Urban Development Planning and development of urban and rural settlements]. Moscow, Gorstroy, 2016. 89 p.

23. Rodionov A.S., Rodionova 0.K. Random Hypernets in Reliability Analysis of Multilayer Networks. Lecture Notes in Electrical Engineering, 2015, vol. 343, pp. 307-315.

24. Rodionov A.S., Rodionova 0.K. Using Random Hypernets for Reliability Analysis of Multilevel Networks. $1^{\text {st }}$ Int. Conf. on Mathematical Methods and Computational Techniques in Science and Engineering (MMCTSE 2014). Ser. Mathematical Methods in Science and Engineering. Athens, Greece, 2014. pp. 119-121.

25. Colbourn Ch.J. The Combinatorics of Network Reliability. New York, Oxford University Press, 1987. 160 p.

26. Martnez S.P., Calvino B.0., Rocco S.M. All-Terminal Reliability Evaluation through a Monte Carlo Simulation Based on an MPI Implementation. European Safety and Reliability Conference: Advances in Safety, Reliability and Risk Management (PSAM 2011/ESREL 2012). Helsinki, 2012. pp. 1-6.

27. Migov D.A., Rodionov A.S. Parallel Implementation of the Factoring Method for Network Reliability Calculation. ICCSA-2014. LNCS. Heidelberg, Springer, 2014. Vol. 8584 (4), pp. 654-664.

28. Nesterov S.N., Migov D.A. Parallel Calculation of Diameter Constrained Network Reliability. PACT-2017. LNCS. Heidelberg, Springer, 2017. Vol. 10421, pp. 473-479.

29. Shooman A.M., Kershenbaum A. Exact Graph-Reduction Algorithms for Network Reliability Analysis. IEEE Global Telecommunications Conference GLOBECOM' 91. New York, IEEEP Press, 1991. pp. 1412-1420.

30. Rodionov A.S. Cumulative Estimated Values of Structural Networks Reliability Indices and their Usage. IEEE Conf. on Dynamics of Systems, Mechanisms and Machines. New York, IEEE Press, 2016. pp. 1-4.

31. Rodionov A.S. Cumulative Estimated Values of Structural Networks Reliability Indices and their Usage. Problems of informatics, 2017, no. 34, pp. 15-24. In Rus.

32. Dorigo M. Swarm Intelligence, Ant Algorithms and Ant Colony Optimization. Reader for CEU Summer University Course «Complex System». Budapest, 2001. pp. 1-38.

Received: 8 November 2018.

\section{Information about the authors}

Gulzhigit Y. Toktoshov, Cand. Sc., researcher, Institute of Computational Mathematics and Mathematical Geophysics of SB RAS.

Anastasiya N. Yurgenson, Cand. Sc., researcher, Institute of Computational Mathematics and Mathematical Geophysics of SB RAS.

Denis A. Migov, Cand. Sc., senior researcher, Institute of Computational Mathematics and Mathematical Geophysics of SB RAS. 